Proceedings

Elsevier Editorial System(tm) for NIMB

Manuscript Draft

Manuscript Number: NIMB_PROCEEDINGS-D-13-00112R2

Title: Combined model of the material excitation and relaxation in swift heavy ion tracks

Article Type: Special Issue: ICACS-25

Section/Category: Special Issue: ICACS-25

Keywords: swift heavy ion track, dynamical structure factor, molecular dynamics, Monte-Carlo simulations, electron-lattice coupling

Corresponding Author: Dr. Alexander Volkov, Ph.D.

Corresponding Author's Institution: National Research Centre Kurchatov Institute

First Author: Sergej A Gorbunov

Order of Authors: Sergej A Gorbunov; Pavel N Terekhin; Nikita A Medvedev, Ph.D.; Alexander Volkov, Ph.D.

Manuscript Region of Origin: RUSSIAN FEDERATION

Abstract: A multiscale approach describing material excitation in the nanometric track of a swift heavy ion (SHI) decelerated in a solid in the electronic stopping regime is presented. This model consists of a combination of three different methods: (a) Monte Carlo simulations of excitation of the electron subsystem of a solid at the femtosecond scale due to scatterings of a SHI and generated fast electrons; (b) a molecular-kinetic approach describing the spatial spreading of electrons after finishing of ionization cascades up to timescales of a hundred femtoseconds; and (c) molecular dynamics simulations of reaction of the lattice on the excess energy transferred from the relaxing electron subsystem at the picosecond time scale. The Dynamic Structure Factor (DSF) formalism links together all these methods. It takes into account effects of spatial and temporal correlations in the atomic system of a target during its interaction with excited electrons in an ion track. For LiF crystals a good agreement is demonstrated between track heating estimated from the experimental data and that predicted by the model.

Suggested Reviewers:

Response to Reviewers: Dear Editors,

> However, his/her decision is "minor revision".

Reviewer \#2: 


\title{
Combined model of the material excitation and relaxation in swift heavy ion tracks
}

\author{
S.A.Gorbunov ${ }^{1}$, P.N.Terekhin ${ }^{2}$, N.A. Medvedev ${ }^{3}, \underline{\text { A.E. Volkov }}^{1,2,4}$ \\ ${ }^{1}$ LPI of the Russian Academy of Sciences, Leninskij pr., 53,119991 Moscow, Russia \\ ${ }^{2}$ National Research Centre 'Kurchatov Institute', Kurchatov Sq. 1, \\ 123182 Moscow, Russia \\ ${ }^{3}$ CFEL at DESY, Notkestr. 85, 22607 Hamburg, Germany \\ ${ }^{4}$ JINR, Joliot-Curie 6, 141980 Dubna, Moscow Region, Russia
}

\begin{abstract}
A multiscale approach describing material excitation in the nanometric track of a swift heavy ion (SHI) decelerated in a solid in the electronic stopping regime is presented. This model consists of a combination of three different methods: (a) Monte Carlo simulations of excitation of the electron subsystem of a solid at the femtosecond scale due to scatterings of a SHI and generated fast electrons; (b) a molecular-kinetic approach describing the spatial spreading of electrons after finishing of ionization cascades up to timescales of a hundred femtoseconds; and (c) molecular dynamics simulations of reaction of the lattice on the excess energy transferred from the relaxing electron subsystem at the picosecond time scale. The Dynamic Structure Factor (DSF) formalism links together all these methods. It takes into account effects of spatial and temporal correlations in the atomic system of a target during its interaction with excited electrons in an ion track. For LiF crystals a good agreement is demonstrated between track heating estimated from the experimental data and that predicted by the model.
\end{abstract}

Keywords: swift heavy ion track, dynamical structure factor, molecular dynamics, Monte-Carlo, electron-lattice coupling

PACS: 72.10.-d, 11.80.La, 72.15.Lh, 72.20.Jv, 61.80.-x, 05.10.Ln, 52.65.Yy

*) a.e.volkov@mail.ru 
model is applied to estimate lattice heating in tracks of $2 \mathrm{GeV} \mathrm{Au}$ and $0.96 \mathrm{GeV} \mathrm{Kr}$ ions in LiF crystals.

The above mentioned models use the cross sections of interaction of fast electrons (and a SHI in the MC) with the electron and atomic subsystems of a target. The simplest but efficient approach describing interaction of incident projectiles with a dynamically coupled system of particles is based on (a) one-particle approximation for the traced projectile; and (b) the assumption of a weak interaction of this projectile with the scattering system (the first Born approximation). In this case, the differential cross section is factored into the cross section describing scattering of a projectile on an isolated particle of the scattering ensemble and a term describing a collective response of this ensemble to the transferred energy and momentum.

At least two realizations of this approach are known. The first one, applied originally to describe scattering of neutrons [7], is based on the formalism of the dynamic structure factor $S(\vec{k}, \omega)$ of the lattice (DSF, $\hbar \vec{k}$ and $\hbar \omega$ are, respectively, the momentum and the energy transferred from a projectile into a scattering system in a collision under consideration, and $\hbar$ is the Plank constant). The second one, commonly used in plasma physics for description of a response of an ensemble of charged particle, is based on the formalism of the complex dielectric function $\varepsilon(\vec{k}, \omega)$ (CDF) [6]. In this case the fluctuation-dissipation theorem [8] states that in a local equilibrium the DSF and CDF formalisms are equivalent (e.g. for electrons $\left.S^{e}(\vec{k}, \omega)=-\left(\hbar k^{2} / 4 \pi^{2} e^{2} n\right) \operatorname{Im} \varepsilon_{e}^{-1}(\vec{k}, \omega)\right)$.

For the case of swift heavy ion impact, the above mentioned approximations are justified for both, (a) an incident ion interacting with the electronic subsystem of a target, and (b) for excited electrons. In this paper, the CDF formalism is used in the Monte Carlo algorithm describing the cross-sections of inelastic scattering of an SHI and electrons. 
The DSF is used to calculate the elastic scattering of electrons, transferring their kinetic energy to the target lattice. MD tracing of atoms enables getting of the transient DSF and, thus, realistic cross-sections of the electron-lattice energy exchange in a relaxing SHI track. The same MD also traces the atomic relaxation and spatial dissipation of the excess energy of the lattice after cooling down of the electron ensemble.

In contrast to previous multiscale approaches of SHI track modeling [9-13], the present one is relying neither on phonon (low excitation) nor on plasma approximation (extremely high excitation) for the description of electron-to-lattice coupling. The DSF/CDF formalism includes automatically both limit cases along with all the possible intermediate states of an irradiated target.

For LiF crystals a good agreement is demonstrated between track heating estimated from the experimental data [14] and that predicted by the model.

\section{Monte Carlo model of SHI penetration and the initial kinetics of the electron ensemble in a SHI track}

In this paper, we use a Monte-Carlo model to describe the excitation of the electronic subsystem of a target. Its physical background and details of the numerics used are described in detail in Refs. $[15,16]$ where this code was tested by a comparison with the results of SHI irradiations of $\mathrm{SiO}_{2}$ and $\mathrm{LiF}$ as well as other MC simulations.

At the first step, the model simulates the passage of an ion and its energy losses which accomplish with the creation of the first generation of highly excited free electrons. The energy transfer during a single projectile-electron interaction is negligible comparing to the kinetic energy of a projectile due to the large difference between the masses of an electron and an SHI. Indeed, for the energy of ions of $11 \mathrm{MeV} / \mathrm{amu}$ (UNILAC accelerator, GSI, Darmstadt) the maximum energy transferred to an electron is $24 \mathrm{keV}$. Therefore the first Born approximation can be used for description of scattering of an SHI on the electronic subsystem 
of a solid. Taking this into account we have improved, in comparison to [15], the crosssections of scattering of an ion by applying the CDF-based formalism [17]. The dielectric function $\varepsilon(\vec{k}, \omega)$ was restored from the experimental data on the optical coefficients of $\mathrm{LiF}$ $[18,19]$.

Propagation of fast electrons generated in the ion track is modeled even-by-event, accounting for the secondary impact ionizations, leaving holes in deep and valence shells, and/or elastic scattering on lattice atoms. Again, we apply the CDF-based cross-sections [17] to describe scattering of these electrons. Indeed, even for solid state concentrations of the electrons, the energy of their pair Coulomb interaction $U_{e-e} \sim e^{2} n_{e}{ }^{1 / 3}$ does not exceed $5 \mathrm{eV}$. In $\mathrm{LiF}$ we stop MC tracing of an electron when its energy falls below $E_{t r}=3 E_{\text {gap }}\left(E_{\text {gap }}=14.6 \mathrm{eV}\right.$ is the band gap) that occurs at $\sim 10 \mathrm{fs}$ after the projectile passage. This threshold corresponds to the minimal electron energy necessary for production of a stable electron-hole pair in LiF [16]. Due to the large difference $E_{t r} \gg U_{e-e}$ we can use the first Born approximation when describing the scattering of such electrons on the electronic subsystem of a target. The same reason results in the one electron approximation for the ensemble of fast electrons.

Created secondary fast electrons are modeled in the same manner. Production of secondary free electrons due to Auger decay of deep holes is also taken into account in the MC model.

The MC procedure is iterated for $\sim 10^{4}$ times to obtain a trustworthy statistical averaging. The averaged spatial distributions of the electron concentration and the energy density are then extracted and used as the initial conditions for further modeling. Figs. $1 \mathrm{a}$ and $\mathrm{b}$ present these initial radial distributions calculated in the layer of thickness of $10 \mathrm{~nm}$ along the ion path after an impact of $2 \mathrm{GeV} \mathrm{Au}$ and $0.96 \mathrm{GeV} \mathrm{Kr}$ ions in $\mathrm{LiF}$.

\section{Spatial spreading of electronic excitations after finishing of ionization cascades}


We stop the MC tracing of electrons when they fall below the cut off energy, as discussed above. Application of the many-particles kinetic equation is a general method to describe the kinetics of the ensemble of interacting low energy electrons. However, the spatial redistribution of excited electrons is the main process in the kinetics of electrons in the vicinity of the ion trajectory at times after finishing of the ionization cascades ( $10 \mathrm{fs}$ after the projectile passage) until the equilibration of the electronics temperature with the lattice temperature. Taking this into account, we use a simplified approach to describe the kinetics of the excited electron ensemble in this time frame.

First, we divide the area around the projectile trajectory in the cylindrical layers of thicknesses larger than the mean free path of electrons. We assume a local equilibrium in the electron ensemble in these layers because of the significant differences between the characteristic times of thermalization of these electrons $\left(\sim 10^{-15} \mathrm{sec}\right)[2]$ and the energy transfer from the electron ensemble to the lattice $\left(>10^{-14} \mathrm{sec}\right)$. Taking into account that the concentration of excited electrons at such times is lower than the solid state one and, moreover, is continuously decreasing due to spatial redistribution, we apply the one particle (gas) approximation to description of the state of the ensemble of electrons.

The parameters of the equilibrium Fermi distribution of electrons in the free-electron approximation, namely the chemical potential and the temperature, can be restored in each cylindrical layer from the current concentration and the average electron energy in a layer [9, 20]:

$$
\begin{aligned}
& n_{g}^{e}=\frac{2 m_{e}^{3 / 2}}{\sqrt{2} \pi^{2} \hbar^{3}} \int_{0}^{\infty}\left[\exp \left(\frac{\varepsilon-\mu_{g}}{k_{B} T_{g}}\right)+1\right]^{-1} \times \sqrt{\varepsilon} d \varepsilon, \\
& E_{g}=\frac{2 m_{e}^{3 / 2}}{\sqrt{2} \pi^{2} \hbar^{3}} \int_{0}^{\infty}\left[\exp \left(\frac{\varepsilon-\mu_{g}}{k_{B} T_{g}}\right)+1\right]^{-1} \times \varepsilon^{3 / 2} d \varepsilon
\end{aligned}
$$


Here $m_{e}$ is the electron mass, $n_{g}^{e}$ is a concentration of electrons, $E_{g}$ is the total energy density of electrons in $g$-th layer. The initial concentrations and the energy of electrons in the layers are taken from the MC calculation.

The difference between the concentrations and average electron velocities in different layers, i. e. their spatial gradients, provide transfer of electrons and their energy between the layers. To describe this transfer the molecular-kinetic method is applied at this stage lasting from the moment when spatial spreading of excited electrons turns into diffusive behavior [9] until the time when the electron temperature equilibrates with the atomic one. In the framework of this approach, each cylindrical layer is divided into a number of small cubes. Due to the equilibrium, the number of electrons $\Delta N_{e}$ escaping from a cube centered in a point $\vec{r}$ trough the grain $\Delta S$ during time $\Delta t$ is defined by

$$
\Delta N_{e}=\frac{1}{6} n_{e}(\vec{r})\left\langle v_{e}(\vec{r})\right\rangle \Delta S \Delta t
$$

Here $n_{e}(\vec{r})$ is the concentration of electrons and $\left\langle v_{e}(\vec{r})\right\rangle$ is their average velocity in the cube. The time step $\Delta t$ was chosen to provide $\left\langle v_{g}\right\rangle \Delta t$ much smaller than the thickness of the layer.

The temporal evolution of the radial distributions of the concentration of electrons and their energy density in the cylindrical layers provides the temporal dependencies of the local equilibrium distribution functions of electrons $f_{\vec{k}}$ in any $g$-th layer.

\section{The model of electron-to-lattice energy exchange in a SHI track.}

\subsection{The DSF based electron-to-lattice energy transfer rate}

Taking into account the one-electron approximation, the energy transfer rate $Q=-d E / d t$ to the lattice due to elastic scattering of electrons is defined by [21]:

$$
Q=\frac{\hbar^{4}}{2 \pi^{3} m_{e}^{2}} \int f_{\vec{k}_{i}}\left(1-f_{\vec{k}_{f}}\right) \omega \frac{k_{i}}{k_{f}} \frac{\partial^{2} \sigma}{\partial \Omega_{f} \partial E_{f}} d \vec{k}_{i} d \vec{k}_{f}
$$


Here $f_{\vec{k}_{i}}$ and $f_{\vec{k}_{f}}, \vec{k}_{i}$ and $\vec{k}_{f}$ are, respectively, the equilibrium distribution functions of electrons and the initial and final wave vectors of an electron; $\Omega_{\mathrm{f}}$ is the solid angle corresponding to the outgoing wave vector $\vec{k}_{f}$, and $E_{f}=\hbar^{2} \vec{k}_{f}^{2} / 2 m_{e}$ is outgoing energy of scattered particle, $\hbar \omega$ is the change of the energy of an electron. Note, that Eq. (3) is a general formula, which can be applied for the case of nonequilibrium distribution functions as well.

Due to the large difference in the masses of an electron and lattice atoms $\left(m_{e} / M_{a} \sim 10^{-}\right.$ ${ }^{5}<<1$, as well as due to a screening effect, the energy transferred to the lattice during a single collision is small in comparison to the electron energy. Therefore, scattering of electrons on the lattice can be described within the first Born approximation [21]. In this case, the differential cross section describing scattering of an electron on the lattice is factored into the cross section of its scattering on an isolated atom and the of the lattice DSF $S(\vec{k}, \omega)$ [7]:

$$
\frac{\partial^{2} \sigma}{\partial \Omega_{f} \partial E_{f}}=|V(\vec{k})|^{2} \frac{m_{e}^{2}}{4 \pi^{2} \hbar^{5}} \frac{k_{f}}{k_{i}} S(\vec{k}, \omega)
$$

Here $\vec{k}=\vec{k}_{f}-\vec{k}_{i}$ is the change of wave vector of scattered electron, $V(\vec{k})$ is the spatial Fourier transform of the interaction potential between an electron and a single atom of a target. We use the Thomas-Fermi screened potential to describe this interaction:

$$
V(r)=\frac{Z_{a t} e}{4 \pi \varepsilon_{0} r} e^{-\frac{r}{L_{S C R}}}, \quad L_{S C R}^{-2}=\frac{m_{e} e^{2}}{\pi^{2} \hbar^{2} \varepsilon_{0}} \int f_{k} d k
$$

where $L_{S C R}$ is a screening length [3], $Z_{a t}$ is the charge of a lattice ion, $\varepsilon_{0}$ is the vacuum permittivity. The DSF is the Fourier transform of the spatial and temporal pair correlation function $G(\vec{r}, t)$ [7] which describes the coupled atomic dynamics in the lattice:

$$
S(\vec{k}, \omega)=\frac{N}{2 \pi} \int d t d \vec{r} e^{i(\vec{k} \vec{r}-\omega t)} G(\vec{r}, t),
$$




$$
G(\vec{r}, t)=\frac{1}{N}\left\langle\sum_{i, j=1}^{N} \int d \vec{r}^{\prime} \delta\left(\vec{r}+\overrightarrow{\vec{R}}_{i}(0)-\vec{r}^{\prime}\right) \delta\left(\vec{r}^{\prime}-\overrightarrow{\vec{R}}_{j}(t)\right)\right\rangle .
$$

Here, the angle brackets denote the quantum mechanical and statistical averaging over the ensemble of eigenstates of the unperturbed Hamiltonian of the scattering system $\left|\psi_{\xi}\right\rangle$, i.e. $\langle x\rangle=\sum_{\xi} \gamma_{\xi} \cdot\left\langle\psi_{\xi}|x| \psi_{\xi}\right\rangle$, where $\gamma_{\xi}$ is a statistical weight of the $\left|\psi_{\xi}\right\rangle$-state. The statistical averaging is made over a volume with sizes larger than the lengths of dynamic correlations in the lattice; $N$ is the number of atoms in this volume.

\subsection{Molecular dynamics model of DSF calculations}

The classical approximation of $G(\vec{r}, t)$ has a simple form [22, 23]:

$$
G(\vec{r}, t)=\frac{1}{N}\left\langle\sum_{i, j=1}^{N} \delta\left(\vec{r}+\vec{R}_{i}(0)-\vec{R}_{j}(t)\right)\right\rangle,
$$

where $\langle\ldots\rangle$ is the statistical averaging over the classical ensemble of the lattice atoms.

Scattering of an electron on a two component system is described by the pair correlation function [24]:

$$
G(\vec{r}, t)=q_{1} q_{1} G_{1-1}(\vec{r}, t)+q_{1} q_{2} G_{1-2}(\vec{r}, t)+q_{2} q_{1} G_{2-1}(\vec{r}, t)+q_{2} q_{2} G_{2-2}(\vec{r}, t)
$$

Here $q_{1}$ and $q_{2}$ are the model charges of the lattice ions (for $\operatorname{LiF} q_{\mathrm{Li}}=1, q_{\mathrm{F}}=-1$ [25]). The

functions $G_{\alpha-\beta}(\vec{r}, t)(\alpha, \beta=1,2)$ describe the spatial and temporal correlations of atoms of $\alpha$ and $\beta$ kinds:

$$
G_{\alpha-\beta}(\vec{r}, t)=\frac{1}{N}\left\langle\sum_{i=1}^{N \alpha} \sum_{j=1}^{N \beta} \delta\left(\vec{r}+\vec{R}_{i}^{\alpha}(0)-\vec{R}_{j}^{\beta}(t)\right)\right\rangle .
$$

where $N=N_{\alpha}+N_{\beta}$. 
These classical forms (8-10) of the pair correlation function can be used in MD simulations. The averaging over time is invoked to obtain the statistically meaningful results [23]:

$$
G(\vec{r}, t)=\frac{1}{N \eta_{T}} \sum_{\delta=1}^{\eta_{T}} \sum_{i, j=1}^{N} \delta\left(\vec{r}+\vec{R}_{i}\left(t_{\delta}\right)-\vec{R}_{j}\left(t_{\delta}+t\right)\right)
$$

Here $\eta_{T}$ is the number of timesteps necessary to obtain a trustworthy statistical averaging. $\eta_{T}$ may differ in calculations for different swift heavy ions (typically $\eta_{T}$ is on the order of $10^{3}$ ). The periodic boundary conditions are used when calculating the correlation functions.

The characteristic time of energy transfer from the relaxing electron subsystem to the lattice is shorter or comparable to the time of atomic vibrations (see below), i.e. the lower limit of the characteristic times of dynamic correlations within the atomic subsystem. Any changes of the DSF are negligible during these times. Therefore, in the present work, the same DSF was used in Eq. (4) at each step of the model before equilibration between the electron and lattice temperatures.

The applied MD uses the Verlet algorithm with the time-step of 0.5 fs for propagating atomic dynamics. For LiF we use the modified interatomic Tosi-Fumi potential [25], well suiting for these crystals. Possible changes in the interatomic potential caused by the excitation of the electronic subsystem (nonthermal melting effects [26]) were not included in the model. Indeed, these processes might occur only in the very center of a track where the level of electron excitation could be sufficient to induce the nonthermal effects [27]. But this high ionization should be kept a sufficiently long time (a hundred of femtoseconds or longer $[26,28])$ to produce structure transformations or to affect considerably the lattice dynamics. Taking into account the short temporal scale ( $10 \mathrm{fs})$ of ionizations decays in the track centre, we assume in this paper that such processes can be neglected in SHI tracks in LiF. 
The same MD code is used to describe relaxation of the excited lattice at time after equilibration of the electron ensemble with the atomic subsystem (see below). It should be emphasized that in this paper MD calculations are stopped at times 1-10 ps, shorter than the characteristic times of changes in the structure (in the simplest case, changes of the density) and/or phase transformations observed for some systems in SHI tracks, because we are concentrating here on the extraction of the parameters of the excited material which form the driving forces for these possible structure changes. Moreover, no changes were observed in the atomic density of bulk LiF irradiated with SHI [14]. Some modifications could be observed on the surfaces of Li crystals after irradiation [14, 29], however, here we do not investigate such surface effects limiting ourselves to the bulk material.

\subsection{Verification of the MD model of DSF calculations.}

The pair-correlation function of the lattice, or the DSF, is a key function determining the cross-sections of electron-to-lattice coupling. Therefore, we take special care for testing the MD code calculating the DSF of the lattice. Liquid aluminum was chosen for this purpose, because there is a wide set of measurements of the structure factor of this system [30-32].

A piece of liquid aluminum consisting of 500 atoms and thermalized at the temperature $\mathrm{T}$ $=943 \mathrm{~K}$ and at zero pressure was simulated. We used a many-body interatomic potential within the Embedded Atom Method (EAM) [33] in these MD simulations of aluminum. The time when the equilibrium occurred was determined by the coincidence of the distribution of the energies of atoms with the Maxwellian one (approx. 2000 MD steps) [21].

Fig. 2 demonstrates a very good agreement between the calculated and measured [31]

geometrical structure factors $S(\vec{k})=S(k)=\int d \omega S(\vec{k}, \omega)$ of liquid aluminum. Presented in Fig.3 comparison of the energy dependence of the calculated DSF with the experimental one 
(the dependences on $\omega$ at the fixed wave vector $\vec{k}$ ) also indicates that the developed MD model gives reasonable values of DSF for realistic systems.

\section{Heating of LiF crystals during relaxation of the electron subsystem in SHI tracks.}

Summarizing above mentioned, we apply the following iterative scheme for quantitative estimation of the spatial and temporal dependencies of the energy transferred into the lattice of LiF during relaxation of the electron subsystem of a crystal in a SHI track.

First, the MC model is used to determine the initial radial distributions of the parameters characterizing the excited ensemble of electrons - the concentration of electrons and their energy. These initial distributions are used to restore the initial local equilibrium distribution functions of electrons at different distances from the axis of the track.

Then, using the DSF formalism, the rates of the energy exchange of the electron subsystem with the lattice in these cylindrical layers are calculated by Eq. (4). After that, complete energy losses of the electronic subsystem in the layers per a time-step are determined taking into account the calculated electron-lattice energy exchange rates there. The energy transferred from electrons to the lattice in a cylindrical layer is distributed among the different species of atoms by increasing their kinetic energy in accordance with their mass fractions.

Next, the molecular-kinetic Eq. (3) supplies with the changes of the concentration and the energy of electrons in the cylindrical layers due to electron diffusion. Then, the equilibrium electron distribution functions corresponding to the updated energy density and the concentration of electrons are restored in each cylindrical layer according to Eq. (2). These new functions are substituted into the next timestep of the modeling, and the procedure is repeated over again starting from the new initial conditions.

The characteristic time of cooling down of the electronic subsystem in the SHI track due to spatial spreading of the excitation is shorter than that of thermalization of the non- 
equilibrium lattice (see Fig.4). Therefore, in this paper we model the "heating" of the lattice by the spatio-temporal field of the kinetic temperature [23, 34]:

$$
\begin{aligned}
& T_{k i n}(\vec{r}, t)=\frac{T_{k i n_{-} \alpha}(\vec{r}, t)+T_{k n_{-} \beta}(\vec{r}, t)}{2}= \\
& =\frac{1}{6 k_{B}\left(Q_{\alpha}-1\right)} M_{\alpha} \sum_{n=1}^{Q_{\alpha}}\left(\vec{v}_{n}-<\vec{v}_{\alpha}>\right)^{2}+\frac{1}{6 k_{B}\left(Q_{\beta}-1\right)} M_{\beta} \sum_{n=1}^{Q_{\beta}}\left(\vec{v}_{n}-<\vec{v}_{\beta}>\right)^{2}
\end{aligned}
$$

Here $k_{B}$ is the Boltzmann constant; $Q_{\alpha}$ and $Q_{\beta}$ are the numbers of atoms of each type in a volume in which the kinetic temperature is defined $\left(Q_{\alpha}=N_{\alpha}^{g}\right.$ and $Q_{\beta}=N_{\beta}^{g}$ for g-th cylindrical layer), $M_{\alpha}, M_{\beta},<\vec{v}_{\alpha}>,<\vec{v}_{\beta}>$ are the masses and average velocities of these atoms.

Fig. 4 demonstrates equilibration between the electron and the kinetic lattice temperatures within times $<100$ fs. Thus, starting from this time we adapt the Born-Oppemheimer approximation, assuming that the electron temperature follows the atomic one i.e. after this time we stop tracing the electrons, only MD modelling the atomic dynamics for tracing further relaxation and spreading of the excess energy through the lattice out from the ion trajectory.

The temporal dependencies of the radial distributions of the kinetic temperatures of LiF lattice in a track of $2 \mathrm{GeV} \mathrm{Au}$ ion and $0.96 \mathrm{GeV} \mathrm{Kr}$ ion are shown in Fig.5. It demonstrates that the largest part of the excess energy transferred into the lattice is contained within the distances $r<5 \mathrm{~nm}$ from the ion trajectory. The maximum heating of the LiF lattice in this area does not exceed $100 \mathrm{~K}$ which is in a very good agreement with the experimental results [14].

\section{Conclusions}

A combined microscopic model describing all the stages of material excitation in the nanometric vicinity of the SHI trajectory is presented. Simulations describe excitation of the electron ensemble of a target by a swift heavy projectile, the subsequent ionization cascades, 
spatial spreading and relaxation of electrons, Auger relaxation of deep holes, energy and momentum transfer from the excited electron subsystem into lattice and subsequent lattice dynamics.

The application of the CDF-DSF formalisms automatically takes into account a possible realization of the various limit cases of dynamic correlations in the electron subsystem as well as in the lattice. The results of application of this model to LiF irradiated with swift heavy ions demonstrate the low upper limit of the lattice heating $(\sim 100 \mathrm{~K})$ in a good agreement with that estimated from the experimental results.

\section{Acknowledgements}

The financial supports from Deutsche Forschungsgemeinschaft (Grant TR 973/1-1) and Helmholtz Association (Grant IK-RU-002) are acknowledged.

\section{References}

1. F.F. Komarov, Physics-Uspekhi, 46 (2003) 1253.

2. A.E. Volkov, V.A. Borodin, Nucl. Instr.Meth. B, 146 (1998) 137.

3. Z. Lin, L.V. Zhigilei, V. Celli, Phys. Rev. B, 77 (2008) 075133.

4. B. Rethfeld, A. Kaiser, M. Vicanek and G. Simon, Phys. Rev. B, 65 (2002) 214303.

5. J. Vorberger, D.O. Gerike, International Symposium on High Power Laser Ablation, AIP Conf. Proc. 1464 (2012) 572.

6. M.W.C. Dharma-wardana, F. Perrot, Phys. Rev. E, 58 (1998) 3705.

7. L. van Hove, Phys. Rev., 95 (1954) 249.

8. H. C. Weissker, J. Serrano, S. Huotari, E. Luppi,M. Cazzaniga, F. Bruneval, F. Sottile, G. Monaco,V. Olevanoand, L. Reining, Phys. Rev. B, 81 (2010) 085104.

9. O. Osmani, N. Medvedev, M. Schleberger, B. Rethfeld, Phys. Rev. B, 84 (2011) 214105.

10. Z. Insepov, M. Terasawa and K. Takayama, Phys. Rev. A 77 (2008) 062901. 
11. A.V. Lankin, I. V.Morozov, G. E. Norman S. A. Pikuz, Jr. and I. Yu. Skobelev, Phys.Rev.E, 79 (2009) 036407.

12. J. Wang, M. Lang, R.C. Ewing and U. Becker, J. Phys.: Condens. Matter, 25 (2013) 135001.

13. S.L. Daraszewicz, D.M. Duffy, Nucl. Instr.Meth. B, 269 (2011) 1646.

14. K. Schwartz, A.E. Volkov, M.V. Sorokin, C. Trautmann, K.-O. Voss, R. Neumann, and M. Lang, Phys. Rev. B, 78 (2008) 024120.

15. N.A. Medvedev, A.E. Volkov, N.S. Shcheblanov, B. Rethfeld, Phys. Rev. B, 82 (2010) 125425.

16. N.A. Medvedev, A.E. Volkov, K. Schwartz and C. Trautmann, Phys. Rev. B 87 (2013) 104103.

17. N. Medvedev, R. Rymzhanov A.E. Volkov, NIMB (2012) submitted.

18. E.D. Palik, Handbook of Optical Constants, (Academic Press, San Diego, 1991).

19. B.L. Henke, E.M. Gullikson, and J.C. Davis. Atomic Data and Nuclear Data Tables 54 (1993) 181-342, http://henke.lbl.gov/optical_constants/atten2.html

20. L.D. Landau., E.M. Lifshitz., Statistical physics vol. 5. part 1 (3ed., Pergamon, 1980).

21. G. Baym, Phys Rev, 135 6A (1964) 1691.

22. R. Aamodt, K.M. Case, M. Rosenbaum and P. F. Zweipel, Phys Rev., 126 (1962) 1165 .

23. M. Rieth, Nano-Engineering in Science and Technology, (World Scientific Publishing Co. Pte. Ltd., USA, 2003).

24. A.B. Bhatia, D.E. Thornton, Phys. Rev. B, 2 (1970) 3004.

25. A.B. Belonoshko, R. Ahuja, and B. Johansson, Phys. Rev. B, 61 (2000) 11928.

26. A. Rousse, C. Rischel et al. Nature, 410 (2001) 65.

27. V.V. Stegailov, Contrib. Plasma Phys., 50 No. 1 (2010) 31. 
28. S. K. Sundaram et al., Nature Mat., 1 (2002) 217.

29. M. Terasawa, Vacuum, 81 (2006) 142.

30. T. Scopigno, U. Balucani, G. Ruocco, and F. Sette, Phys. Rev. E, 63 (2000) 011210.

31. D.J. González, L.E. González, J.M. López, and Malcolm J. Stott, J. Chem. Phys., 115 (2001) 2373.

32. J.M. Stallard, C. M. Davis, Phys. Rev. A, 8 (1973) 368.

33. X.Y. Liu, F. Ercolessi, J.B. Adams, Modeling Simul. Mater. Sci. Eng., 12 (2004) 665.

34. J.G. Powles, G. Rickayzen, D.M. Heyes, Mol. Phys., 103 (10) (2005) 1361. 


\section{Figure Captions}

FIG. 1. The radial distributions of the electron concentration (a), and the energy density of electrons (b), in tracks of $\mathrm{Au} 2 \mathrm{GeV}$ and $\mathrm{Kr} 0.96 \mathrm{GeV}$ ions in $\mathrm{LiF}$.

FIG. 2. The geometrical structure factor of liquid aluminum. Red squares show the results of the calculations made in this paper, white circles are the results of the experiment on neutron scattering, black circles are the data from X-ray scattering [31].

FIG. 3. The DSF of liquid aluminum. Red squares are the results of our calculations, the black line is the experimental data from X-ray scattering [30].

FIG. 4. The temporal dependencies of the radial distributions of the temperature of electrons and the kinetic temperatures of the $\mathrm{LiF}$ lattice in the cylindrical layer $1 \mathrm{~nm}<R<1.5$ $\mathrm{nm}$ at different times after the passage of (a) a gold ion with the energy $2 \mathrm{GeV}$ and (b) a krypton ion with the energy $0.96 \mathrm{GeV}$

FIG. 5. The kinetic temperature of atoms of $\mathrm{LiF}$ in a track of the (a) $2 \mathrm{GeV}$ gold ion and (b) $0.96 \mathrm{GeV}$ krypton ion as the functions of time. 


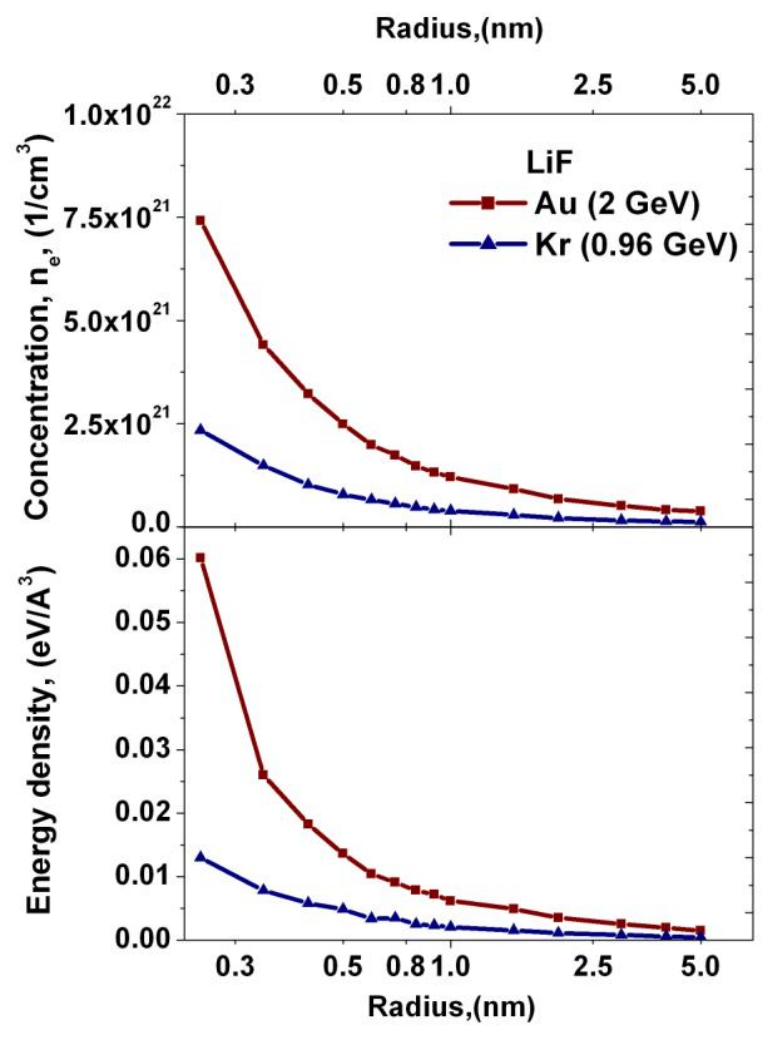

Fig.1 


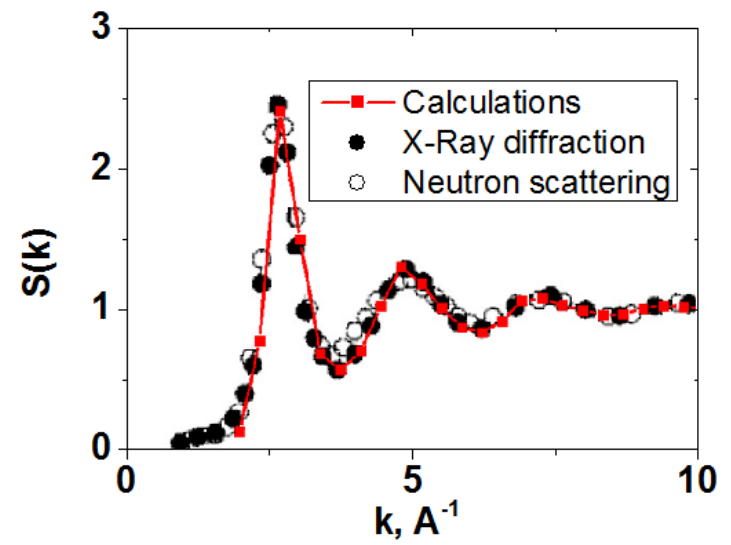

Fig. 2 


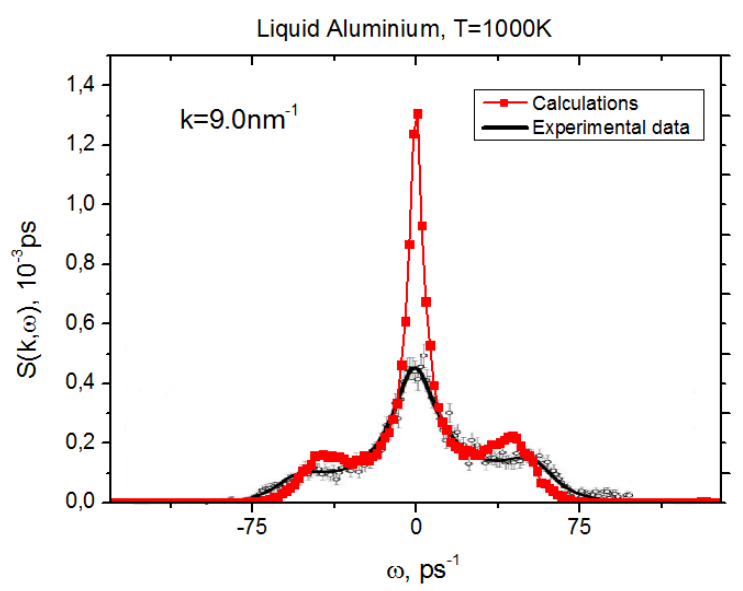

Fig. 3 


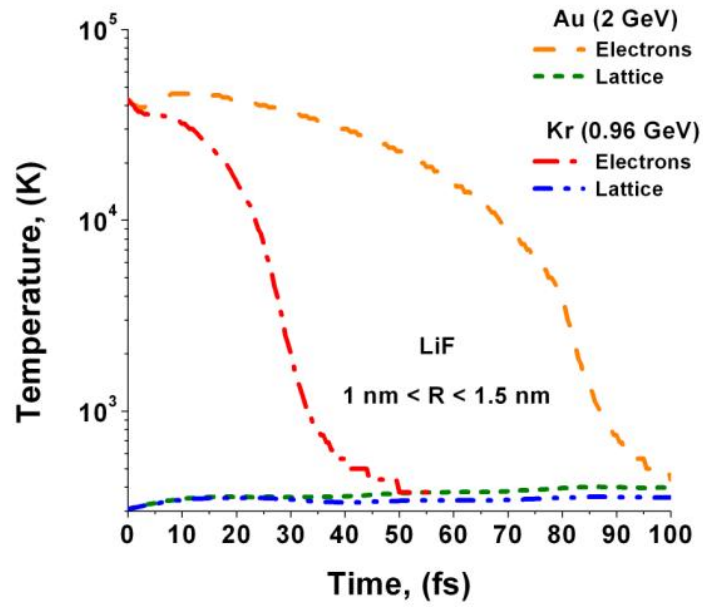

Fig. 4 


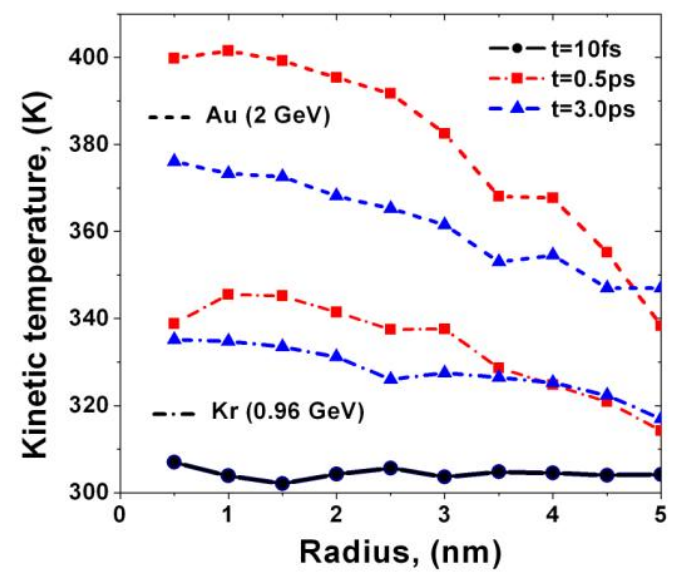

Fig. 5 